\title{
Generation of definitive endoderm from human embryonic stem cells cultured in feeder layer-free conditions
}

\section{CharlesC King, Gillian M Bettie Ana D Loper \& AlbertoHaydk}

${ }^{\dagger}$ Author for correspondence University of California, Whittier Institute, D epartment of Pediatrics, San D iego, 9500 Gilman $D$ rive, Center for $M$ olecular Genetics, Room 131, La Jolla, CA 92037-0634, USA Tel.: +1 858534 1419; Fax: +1 858534 1422;

E-mail: chking@ucsd.edu

Keywords cell culture, definitive endoderm, human embryonic stem cells, Neu5G C

future, part of fs?

\begin{abstract}
Purpose: The purpose of these studies was twofold: to reduce the level of nonhuman, potentially immunogenic sialic acid N-glycolylneuraminic acid (Neu5Gc) in human embryonic stem cells (hESCs) through culture of the cells in the absence of feeder layers; and to determine whether directed differentiation was preserved under these conditions, that is, using exclusively human-derived products. Methods: Using a technique developed in our laboratory to culture hESCs in the absence of feeder layers, all nonhuman cell culture reagents were replaced with recombinant or human-derived reagents. The level of the nonhuman sialic acid (Neu5Gc) was measured by high-performance liquid chromatography and monitored over many passages. Subsequently, the cells were subjected to in vitro differentiation into definitive endoderm by lowering the serum concentrations and elevating the amount of activin A. Results: Under standard tissue culture conditions using mouse and other animal products, the basal levels of Neu5Gc were measured between 7 and $10 \%$. After the cell culture reagents were changed to all human products, Neu5Gc levels decreased steadily before leveling below $2 \%$. Upon initiation of the differentiation protocol under these cell culture conditions, we observed robust endoderm formation, as measured by fluorescence-activated cell sorting analysis and the appearance of mRNA for markers of definitive endoderm (Sox17, CXCR4, Goosecoid and FoxA2). Conclusion: Consistent with other findings, elimination of nonhuman products in cell culture of hESCs decreases the levels of nonhuman and potentially immunogenic sialic acid levels. Furthermore, our studies demonstrate that in this feeder layer-free system, hESCs undergo directed differentiation into definitive endoderm.
\end{abstract}

As human embryonic stem cells (hESCs) become a more common research tool, the need for a better understanding of the basic biochemistry behind maintenance of pluripotency and differentiation of these cells becomes more important. 0 ver the past few years, our laboratory and others have begun to understand how activin-mediated signaling maintains pluripotency in the absence of contaminating mouse feeder layers, allowing advances in the analysis of global transcription and proteome analysis of hESC s [1-5].

A recent report describes the incorporation of the nonhuman, and possibly immunogenic, sialic acid $\mathrm{N}$-glycolylneuraminic acid ( $\mathrm{N}$ eu5G C) from animal-derived tissue culture products on the surface of cultured hESC $s$ [6]. The presence of $\mathrm{N}$ eu5G $\mathrm{c}$ on $\mathrm{hESC} s$ diminishes enthusiasm for therapeutic use of the cells for the treatment of disease owing to fears of xenogenic rejection through an immune response. $O$ ne possible solution to the problem is to develop and culture new hESC lines on human feeder layers and exclusively human-derived culture products. Another is to determine whether Neu5G c levels can be decreased in existing $h E S C$ lines through manipulation of cell culture conditions.

In this report, we demonstrate that culture of hESC $s$ in the absence of contaminating animal products is associated with a decrease in $\mathrm{Neu} 5 \mathrm{G} \mathrm{c}$ levels. Additionally, we show for the first time that directed differentiation of hESC $s$ into definitive endoderm can occur in the absence of feeder layers.

Materials \& methods

hESC culture

Initial culture of the hESC line H SF 6 on feeder layers was performed on mitomycin $\mathrm{C}$-treated CF 1 mouse feeder layers at $37^{\circ} \mathrm{C}, 5 \% \mathrm{CO}_{2}$ in DSR (high-glucose DMEM-containing knockout serum replacer, glutamine, nonessential amino acids, $0.1 \mathrm{mM} \quad \beta$-mercaptoethanol) media, as previously described [7]. Cells were transferred to feeder layer-free conditions by plating on dishes coated with laminin $(20 \mu \mathrm{g} / \mathrm{ml}$, Chemicon, MA, USA [101]). Three different feeder layer-free culture conditions of hESC s were used in this study. First, hESCs 
were cultured under our standard feeder layerfree cell culture conditions in the presence of conditioned medium (CM ) from mouse embryonic fibroblasts (mEFs) supplemented with $10 \mathrm{ng} / \mathrm{ml}$ basic fibroblast growth factor (bFGF2; Preprotech Inc., NJ, USA [102]). Second, hESC $s$ were also grown in the presence of $D S R+$ knockout [KO] serum + ANK (A: recombinant activin $A, 50 \mathrm{ng} / \mathrm{ml} ; \mathrm{K}$ : human recombinant keratinocyte growth factor [KGF], $50 \mathrm{ng} / \mathrm{ml}$, both from Preprotech; and N : nicotinamide [NIC], $10 \mathrm{mM}$ from Sigma Corp., M O, USA). Finally, hESC s were also grown in DSR containing normal human serum in the presence of ANK. M edium was changed every other day and cells were passaged weekly at 1:3 or 1:4 dilution by gentle treatment with $1 \mathrm{mg} / \mathrm{ml}$ collagenase IV (G ibco BRL, CA, U SA) for $5 \mathrm{~min}$, followed by scraping.

\section{Sialic acid measurements}

Sialic acids were released from cells by mild acidic treatment pf cells followed by derivatization with 1,2 diamino-4,5 methylene dioxybenze and analysis by high-performance liquid chromatography (H PLC), as previously described [8].

\section{Differentiation protocol}

To generate definitive endoderm, hESCs grown in DSR containing normal human serum and AN K, as described previously, were switched from DSR to RPM I media containing $100 \mathrm{ng} / \mathrm{ml}$ activin $A$ in the absence of serum. After 1 day, the medium was changed to RPM I containing $100 \mathrm{ng} / \mathrm{ml}$ activin $A$ in the presence of $0.2 \%$ serum and incubated for another $48 \mathrm{~h}$. Subsequently, cells were lysed and $\mathrm{mRNA}$ was prepared.

\section{FACS analysis}

hESC s were originally sustained in D M EM /F 12 with 20\% knockout serum replacement (KO-SR), $4 \mathrm{ng} / \mathrm{ml} \mathrm{bFGF}$ and $10 \mathrm{ng} / \mathrm{ml}$ activin A. At passage, 61 cells were transferred in condition media from $\mathrm{mEFs}$ supplemented with $8 \mathrm{ng} / \mathrm{ml} \mathrm{bFGF}$ on BD growth factors-reduced matrigel and split every 4-5 days. D ifferentiation was started when cells reached confluence. After a brief wash with RPM I, cells were differentiated for 4 days in media containing activin A $(100 \mathrm{ng} / \mathrm{ml})$ and a $0.2 \%$ human serum. O n day $1, \mathrm{hESC} s$ were first exposed for $24 \mathrm{~h}$ to Wnt3A $(25 \mathrm{ng} / \mathrm{ml})$ and activin A $(100 \mathrm{ng} / \mathrm{ml})$ in RPM I media. For the next 2 days, the cells were incubated with $0.2 \%$ human $A B$ serum and activin A (100 ng/ml). On day 4, the concentration of human $A B$ serum was increased to a final concentration of $2 \%$, while the activin $A$ concentration was held constant.

At day 4, cells were washed with phosphatebuffered saline (PBS) and dissociated with 0.05\% trypsin/EDTA (Invitrogen, CA, USA). Cells were washed twice, pelleted and resuspended in normal human serum to block nonspecific antibody binding. Cells were labeled with CXCR4-PE (R\& D Systems, M N , USA) at $20 \mu \mathrm{l}$ for $0.5 \times 10^{6}$ cells for $45 \mathrm{~min}$ on ice. Cells were washed twice and resuspended in $200 \mu \mathrm{l}$ PBS buffer. C ells were analyzed using a BD FacsScan instrument and CellQ uest software (Cytometri Research LLC, CA, USA).

\section{Reverse transcription-PCR}

RN A was purified using the RN easy minikit, including $D N$ ase treatment ( $Q$ iagen, CA, USA [103]) and reverse transcribed using avian myeloblastosis virus with $3.2 \mu \mathrm{g}$ of random primer (Roche, IN, USA [104]) and $1 \mu \mathrm{g}$ of total RN A in a reaction volume of $20 \mu$. A total of $1 \mu$ of $C D N A$ was used for each $P C R$ reaction in a total volume of $50 \mu \mathrm{l}$. The following primers were used: Sox17, forward primer 5'GGCGCAGCAGAAT CCAGA3', reverse primer, 5'CCACGACTTGCCCAG-CAT 3'; CXCR4, forward primer 5'CACCG CATCTGG-AGAACCA3', reverse primer 5'GCCCATT TCCTCGGTGTAGTT 3'; FoxA2, forward primer 5'GGGAGCGG TGAAGATGGA3', reverse primer 5'TCATGTTGCT-CACGGAGGAGTA3'; Goosecoid forward primer 5'GAGGAGAAAGTGGAG TCTGGTT3' and reverse primer 5'CTCT GATGAGGA-CCGCTTCTG 3'.

The PCR products were loaded onto a $1.2 \%$ agarose gel, run at 100 volts and and stained with ethidium bromide.

\section{Results}

We have previously developed a protocol to culture hESC $s$ in the absence of mouse feeder layers [1]. Accordingly, the cells were grown on laminin-coated tissue culture dishes in the presence of D SR media supplemented with AN K, a mixture of growth factors containing recombinant human activin $A, N I C$ and recombinant human KGF. Starting with these cell culture conditions, we wanted to determine whether we could adjust our cell culture conditions and lower the amount of $\mathrm{Neu} 5 \mathrm{G} c$ expressed on cultured hESC s. 
Figure 1. Total levels of Neu5Gc in human embryonic stem cells are reduced upon culture in the absence of animal products.

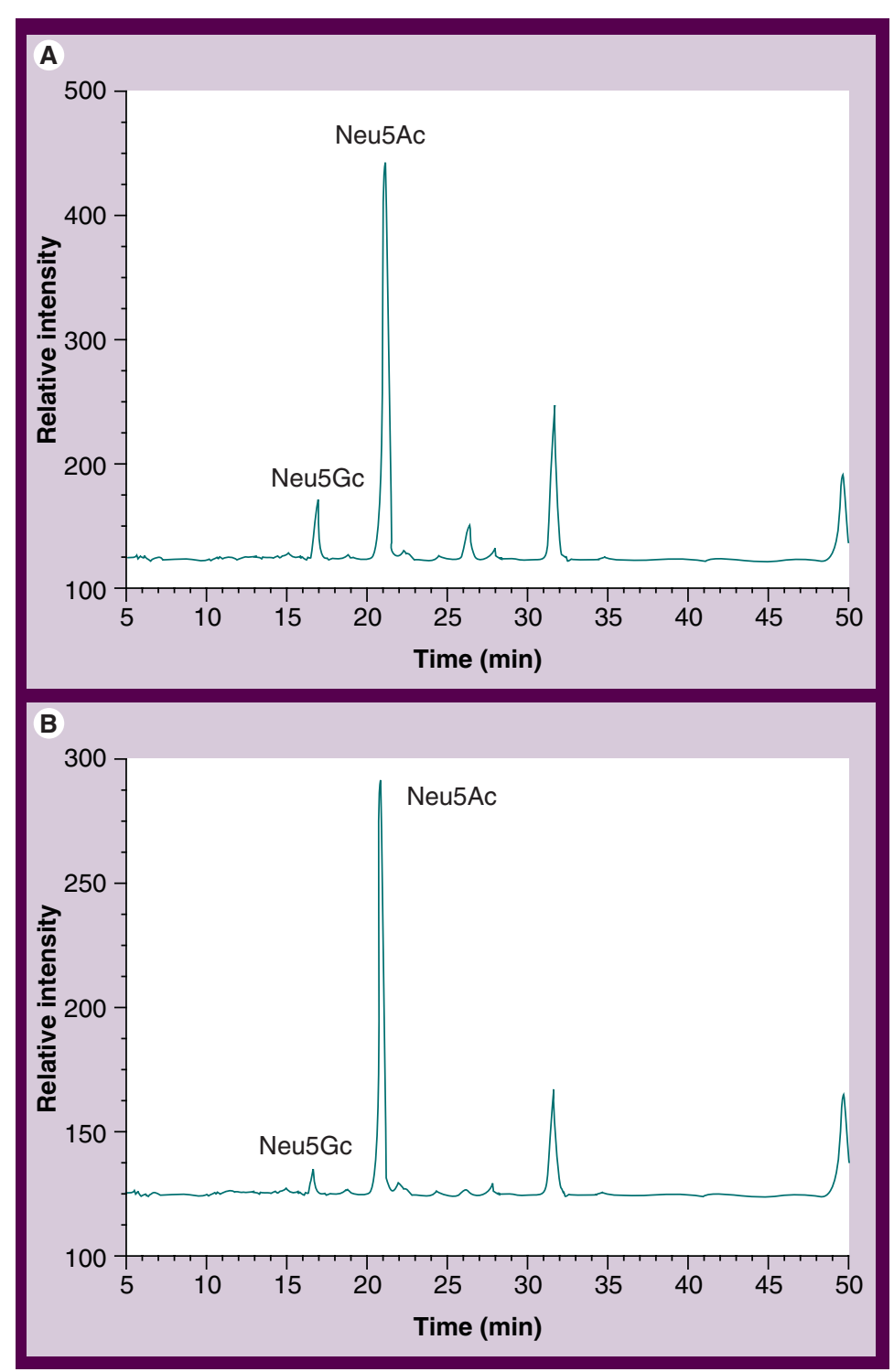

Sialic acids from washed human embryonic stem cells (hESCs) were extracted in mild acid and derivatized with 1,2-diamino-4,5-methylene dioxybenzene and analyzed by high-performance liquid chromatography. The peaks corresponding to Neu5Gc and Neu5Ac are labeled. (A) hESC s cultured in the presence of DSR media supplemented with serum derived from mouse.

(B) hESC s after 4 weeks in culture with exclusively human products.

In initial experiments, we wanted to determine whether the total amount of $\mathrm{Neu} 5 \mathrm{Gc}$ could be reduced when $\mathrm{hESC} s$ were cultured under feeder layer-free conditions in the presence of human serum. Both Neu5G c and the ubiquitous sialic acid $\mathrm{N}$-acetylneuraminic acid ( $\mathrm{N}$ eu5Ac) content on hESC $s$ was measured by HPLC [8]. Under standard hESC culture conditions [1], 908.8 pmols of $\mathrm{N}$ eu $5 \mathrm{G} \mathrm{c}$ were detected per microgram of total protein (pmol/ $/ \mathrm{gg}$ ) by H PLC (Figre1A). After
4 weeks in DSR supplemented with ANK and human serum, the amount of $\mathrm{Neu} 5 \mathrm{G}$ c dropped fivefold to 182.4 pmols (Figre1B), suggesting that a change in cell culture conditions could dramatically alter Neu5G c content. In control experiments, human laminin al one was not identified as a source of N eu5GC (data not shown). Taken together, these results indicate that removal of nonhuman cell culture products reduces $\mathrm{N}$ eu $5 \mathrm{GC}$ expression on $\mathrm{hESC} s$ after multiple passages.

$\mathrm{N}$ ext, we wanted to systematically examine hESC culture conditions to determine which components contributed to $\mathrm{Neu5G}$ c expression on hESC $s$ and to optimize cell culture conditions. To address these questions, H SF 6 cells were grown under three different conditions for up to six passages and membranebound Neu5GC and $\mathrm{N}$ eu5Ac content was measured by H PLC. Cells were passaged weekly and a portion from each passage was retained for protein determination and measurement of sialic acid content. In all experiments, the picomoles of $\mathrm{N}$ eu $5 \mathrm{G} \mathrm{c}$ were normalized to total proteins levels (Figre2). In the first set of experiments, cells were grown in CM from mouse fibroblast feeder layers supplemented with human bFGF and ANK (dark bars). This conditioned media D SR contains growth factors released from mouse fibroblasts and commercially avai lable KO SR (GibCo). The normalized amounts of $\mathrm{Neu} 5 \mathrm{GC}$ remained relatively constant over the course of the experiments, with a range of 6.3 to $9.9 \% \mathrm{Neu} 5 \mathrm{G} \mathrm{c}$ and an average of $7.9 \% \mathrm{~N}$ eu $5 \mathrm{G} \mathrm{C}$.

In the second set of experiments, HSF6 cells were incubated in complete DSR media supplemented with ANK (red bars). Again, this media contained potential nonhuman sialic acids derived from serum. 0 ver five passages during a 5 -week period, the amount of $\mathrm{N}$ eu $5 \mathrm{G} \mathrm{c}$ was again relatively constant, ranging from 7.7 to $10.3 \%$, with an average of $8.5 \% \mathrm{Neu} 5 \mathrm{Gc}$. In the final experimental condition, H SF 6 cells were grown in DSR media supplemented with normal human serum and AN K to maintain pluripotency. After the initial passage, the amount of $\mathrm{Neu} 5 \mathrm{GC}$ dropped from 10.3 to $6.3 \%$. Subsequent passages of cells in the same media saw a continued drop in the amount of $\mathrm{N}$ eu $5 \mathrm{Gc}$ to $2 \%$, where it stabilized (blue bars). These results indicate that the amount of $\mathrm{N}$ eu5G c can be reduced through the elimination of animal products in tissue culture conditions. O ne potential source of $\mathrm{N}$ eu $5 \mathrm{G}$ c contamination, the KO-SR, was tested and found to have a consistently low, but detectable, level of sialic acid. However, the amount detected was lower than the $2 \%$ observed in hESC s. 


\section{Figure 2. Analysis of N-glycolylneuraminic acid content of human embryonic stem cells grown under three different culture conditions.}

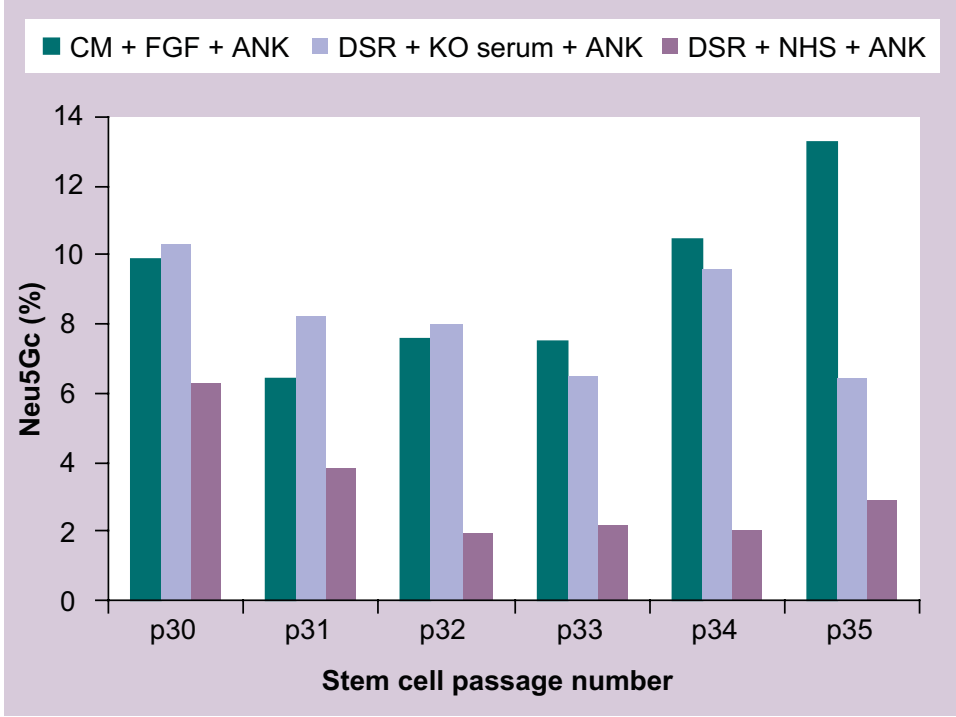

Human embryonic stem cells were grown under feeder layer-free conditions in the presence of CM-mouse + FGF + ANK (green bars), DSR + KO serum + ANK (red bars), or in DSR + NHS + ANK for four passages (blue bars). During each passage, a portion of the cells was removed for protein determination (BCA assay, Pierce) and for high-performance liquid chromatography analysis of Neu5Ac and Neu5Gc.

ANK: Activin, nicotinamide, keratinocyte growth factor; CM: Conditioned media; FGF: Fibroblast growth factor; KO: Knockout; NHS: Normal human serum. generated using the Qiagen RN easy kit, and CDNA was prepared by reverse transcription using iScript CD N A Synthesis K it (Bio-Rad, CA, USA), and reverse transcription-PCR was performed as previously described [4]. Levels of the definitive endodermal markers Sox17, FoxA2, Goosecoid and CXCR4 were quantified (Figre3). Consistent with results previously published by D 'Armour et al. [4], hESC $s$ grown on mEFs in the presence of high activin $A$ levels showed a significant increase in Sox17 and FoxA2 transcript levels. In these experiments, neither the Goosecoid nor the CXCR4 levels were significantly different from the control cells grown on mEFs in the absence of activin A. A significant increase in message for Sox17, F oxA2, G oosecoid and CXCR4 was observed when hESC s were cultured on laminin under conditions previously demonstrated to reduce $\mathrm{N}$ eu5G c levels. These results demonstrate that reduced nonhuman sialic acid levels and hESC culture in feeder layer-free conditions do not interfere with the endodermal differentiation program.

\section{Discussion}

$\mathrm{H}$ ere, we describe the reduction of the nonhuman sialic acid N eu5Gc through the elimination of animal-derived cell culture products. Specifically, much of the Neu5Gc is associated with the serum used during culture, not with the laminin or recombinant protein cocktail used to maintain pluripotency.

During the preparation of this report, other investigators have helped to establish a strong foundation for the reduction of $\mathrm{N}$ eu5G c levels through changes in cell culture conditions $[10,11]$. Heiskanen et al. published a report demonstrating that $\mathrm{Neu} 5 \mathrm{Gc}$ levels could be decreased in both mesenchymal and hESC $s$ using a mass spectrometry and immunohistochemical approach [10]. Cell death resulting from compliment-mediated targeting was demonstrated to be reduced upon removal of hESC $s$ from $\mathrm{mEFs}$ [12], although the results of these experiments have been called into question [13]. O ur results are similar to those obtained by $\mathrm{N}$ asonkin et al., who observed substantial decreases in N eu5G c $(<1 \%)$ during neural differentiation using media composed of B27/N 2/noggin [9]. Taken together, these studies provide strong evidence that culture of $\mathrm{hESC}$ s on animal feeder layers is a significant source of $\mathrm{N} \mathrm{eu} 5 \mathrm{G} \mathrm{c}$ and stem cell death, but that the nonhuman sialic acid levels can be decreased through the manipulation of cell culture conditions. 
Figure 3. Decreased levels of $\mathrm{N}$-glycolylneuraminic acid do not impair human embryonic stem cell differentiation into definitive endoderm.

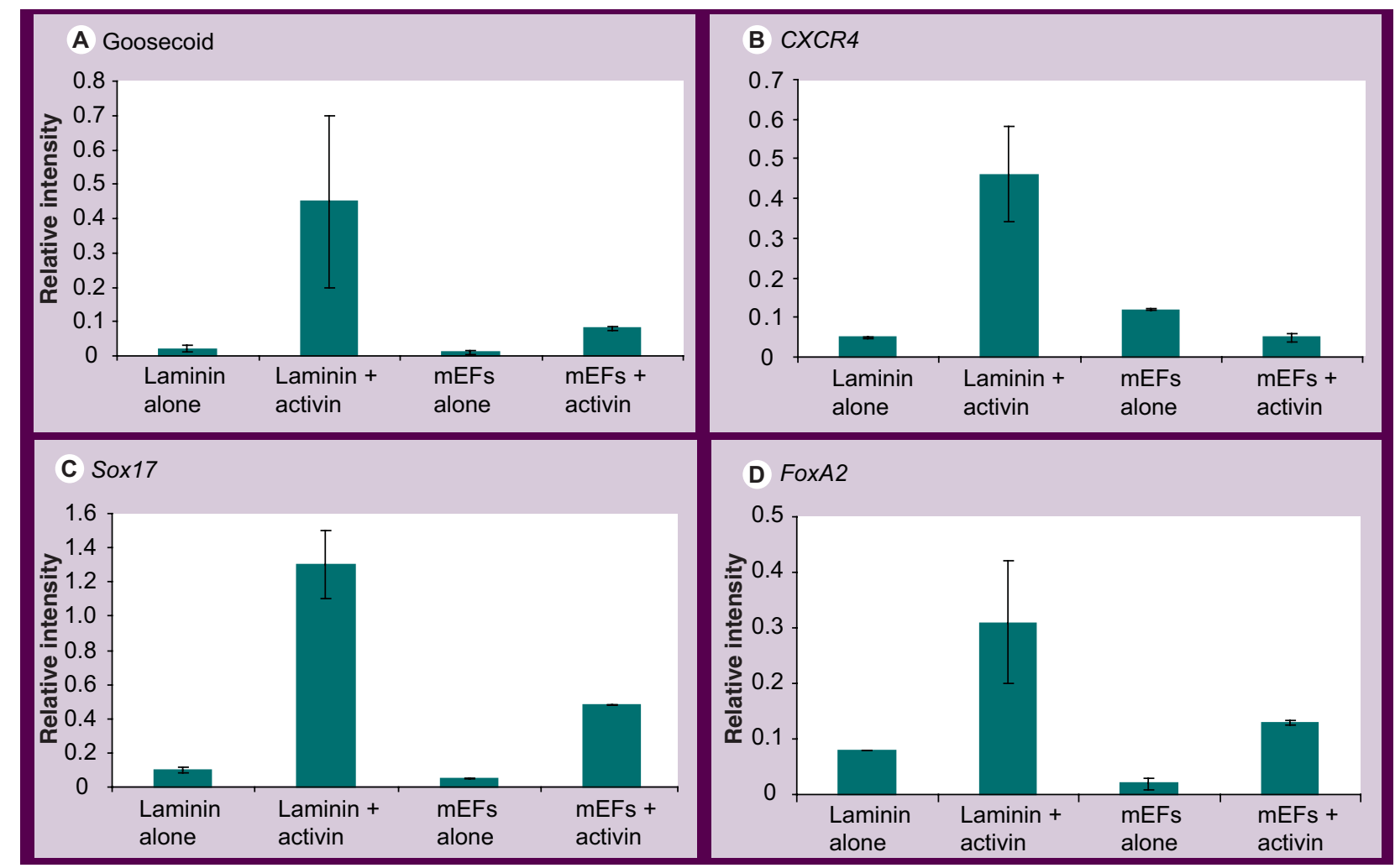

HSF6 cells were either plated on laminin or on mouse embryonic fibroblast feeder layer in the presence or absence of $100 \mathrm{ng} / \mathrm{ml}$ activin A. After 5 days, cells were harvested for quantitative PCR analysis, as previously described [3].

mEF: Mouse embryonic fibroblast.

We also demonstrate that hESCs can undergo directed differentiation to definitive endoderm lineage, when cultured under feeder layer-free conditions. Differentiation occurs whether cells are grown under standard conditions (DSR + KO serum + ANK; data not shown), or in the presence of exclusively human/recombinant-derived cell culture products. Interestingly, cells grown in the absence of feeder layers showed a stronger expression of definitive endodermal markers. Goosecoid and CXCR4 receptor message RNA was increased fourfold, while Sox17 and FoxA2 message RN A was increased threefold over cells differentiated on mEFs. Whether this represents a more robust expression of markers or larger population of cells undergoing differentiation is currently under investigation.

\section{Executive summary}

- The therapeutic use of human embryonic stem cells (hESCs) may be complicated by the presence of the nonhuman and potentially immunogenic sialic acid N-glycolylneuraminic acid (Neu5Gc).

- Changing cell culture conditions of hESCs to a feeder layer-free system containing exclusively recombinant- and human-derived products decreased the detectable amount of Neu5Gc fivefold.

- The culture of cells in animal serum appears to be a major source of Neu5Gc.

- hESCs were demonstrated to undergo directed differentiation to form definitive endoderm when grown under feeder layer-free conditions in the presence of human serum.

- The appearance of markers of definitive endoderm (Goosecoid, CXCR4 receptor, FoxoA and Sox17) was more robust than cells cultured on feeder layers, suggesting that feeder layer-free conditions may be a better system for differentiation of hESC $s$ into definitive endoderm. 


\section{RESEARCH ARTICLE - King, Beattie, Lopez \& H ayek}

\begin{abstract}
Acknowledgements
We gratefully acknowledge the help of I va Afrikanova on the fluorescence-activated cell sorting analysis.

Financial \& competing interests disclosure Thisstudy was supported by the Larry L H illblom Foundation. The authors have no other relevant affiliations or financial involvement with any organization or entity with a financial interest in or financial conflict with the subject matter or materials discussed in the manuscript apart from those disclosed.
\end{abstract}

$\mathrm{No}$ writing assistance was utilized in the production of this manuscript.

\section{Ethical conduct of research}

The authors state that they have obtained appropriate institutional review board approval or have followed the principles outlined in the Declaration of $\mathrm{H}$ elsinki for all human or animal experimental investigations In addition, for investigations involving human subjects, informed consent has been obtained from the participantsinvolved.

\section{Bibliography}

1. Beattie GM , Lopez AD, Bucay N et al.: Activin A maintains pluripotency of human embryonic stem cells in the absence of feeder layers. Stem Cells 23, 489-495 (2005).

2. James $D$, Levine AJ, Besser $D$, H emmati-Brivanlou A: TGF $\beta$ /activin/nodal signaling is necessary for the maintenance of pluripotency in human embryonic stem cells. D evelopment 132(6), 1273-1282 (2005).

3. Vallier $L$, Alexander $M$, Pedersen RA: Activin/nodal and FGF pathways cooperate to maintain pluripotency of human embryonic stem cells. J. Cell Sci. 118(Pt 19), 4495-4509 (2005).

4. D 'Amour KA, Agulnick AD, Eliazer S et al.: Efficient differentiation of human embryonic stem cells to definitive endoderm. $\mathrm{N}$ at. Biotechnol. 23(12), 1534-1541 (2005).

5. Xiao L, Yuan X, Sharkis SJ : Activin A maintains self-renewal and regulates fibroblast growth factor, $\mathrm{W}$ nt, and bone morphogenic protein pathways in human embryonic stem cells. Stem Cells 24(6), 1476-1486 (2006).
6. M artin MJ, M uotri A, Gage F et al.: $H$ uman embryonic stem cells express an immunogenic nonhuman sialic acid. $N$ at. M ed. 11, 228-232 (2005).

7. Humphrey RK, Beattie GM , Lopez AD et al.: $M$ aintenance of pluripotency in human embryonic stem cells is ST AT 3 independent. Stem Cells 22(4), 522-530 (2004).

8. Tangvoranuntakul P, Gagneux P, D iaz S et al.: H uman uptake and incorporation of an immunogenic nonhuman dietary sialic acid. Proc. N atl Acad. Sci. U SA 100(21), 12045-12050 (2003).

9. N asonkin IO, Koliatsos VE: N onhuman sialic acid $\mathrm{N}$ eu $5 \mathrm{G}$ c is very low in human embryonic stem cell-derived neural precursors differentiated with B27/N 2 and noggin: implications for transplantation. Exp. N eurol. 201, 525-529 (2006).

10. H eiskanen A, Satomaa T, Tiitinen $\mathrm{S}$ et al.: $\mathrm{N}$-glycolylneuraminic acid xenoantigen contamination of human embryonic and mesenchymal stem cells is substantially reversible. Stem Cells 25, 197-202 (2007).
11. Ludwig TE, Levenstein M E, Jones JM et al.: D erivation of human embryonic stem cells in defined conditions. $\mathrm{N}$ at. Biotechnol. 24, 185-187 (2006).

12. Cerdan $C$, Bendall SC, Wang $L$ et al.: Complement targeting of nonhuman sialic acid does not mediate cell death of human embryonic stem cells. N at. M ed. 12, 1113-1114 (2006).

13. M artín M, M uotri A, Gage FH, Varki A: Complement targeting of nonhuman sialic acid does not mediate cell death of human embryonic stem cells. $N$ at. M ed. 12, 1115 (2006).

\section{Websites}

101. M illipore www.chemicon.com

102. Peprotech www.peprotech.com

103. Q uiagen: sample and assay technologies www.qiagen.com

104. Roch www.roche-applied-science.com 